\title{
Influence Mechanism of Bilili Gourmet Videos on User Interaction Intention
}

\author{
Gai Wenxin ${ }^{1, a}$

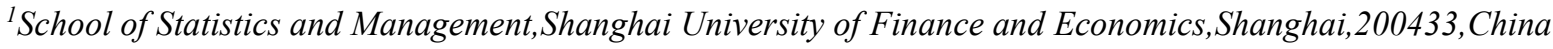 \\ aEmail:xyjtzzbliu@163.com
}

\begin{abstract}
Bilibili has a unique user interaction mechanism. From an empirical point of view, this paper conducted in-depth interviews with users watching Bilibili food videos, putting forward the research hypothesis and model of this paper, and designed a questionnaire, analyzed the influencing factors of user interaction intention. It found that the information emotional and symbolic value and interaction degree of food videos will significantly positively affect user interaction intention. Combined with the research process and results, this paper puts forward some management suggestions for food uploaders, we media platforms and users.
\end{abstract}

Keywords : Bilibili, Food Vedios, User Interaction

\section{INTRODUCTION}

BiliBili barrage network (referred to as Bilibili) is a video website based on UGC. The content created by the uploader (the user who publishes the video) accounts for $90 \%$ of the contributions. The user is not only the producer of the content, the participant of collective creation, but also the consumer and disseminator of the content [1]. Until August 2, 2021, there are 59 uploaders in food category among the uploaders in Bilibili with more than 1 million fans [2]. As a traditional video production content, food videos are loved by the majority of viewers in BiliBili. Based on this, this paper attempts to explore the impact of food videos on user interaction in BiliBili, which can not only provide reference for video creators, but also be of great significance for the long-term development of traffic platform.

\section{RELATED CONCEPTS AND LITERATURE REVIEW}

In 2012, "A Bite of China 1" set off a wave of delicious food in China, which stimulated the people's enthusiasm for watching and tasting delicious food. In the field of short video, food has always been a content type with high playback volume. Some food short video we media are gradually moving towards scale and branding. Food videos are infectious and remind viewers of life [3]. Most scholars' research focuses on the content and expression forms of video. For example, Tang Menglin
(2018) studied the content characteristics of food video [4], Huang Lili (2019) studied the symbolic meaning construction of short food videos in the context of consumer culture [5].

"Interaction" generally refers to the information exchange process between man and all things in nature, indicating the interaction and influence between them [6]. Luo Huanyi (2015) defined video interaction as the process and result of mutual exchange and influence between various forms and levels of online video producers and consumers [7]. Wang Xiwei et al. (2017) pointed out that user information interaction in the new media environment includes the behavior of users using the new media platform to search, browse and disseminate digital information, and exchange information with each other such as online forwarding, comments, likes and sharing [8]. In this paper, user interaction refers to the behavior of users like and comment on video in the process of receiving video information, which is conducive to the platform to push according to the preferences of different users.

\section{MODEL CONSTRUCTION AND ASSUMPTIONS}

\subsection{DEPTH INTERVIEW}

In this interview, open-ended questions were used, and the content of answers was not strictly limited, so interviewees can give free play. This interview mainly 
focuses on "What kind of food videos do you usually watch?" and "What kind of food videos will make you willing to like", and then encodes the answers, and finally puts forward five variables that food videos affect user interaction, information value, entertainment value, emotional value, symbolic value and interaction degree. Details of open coding are showed in Table 1.

Table 1 Open Coding Process

\begin{tabular}{|c|c|c|}
\hline Examples & Conceptualization & Categorization \\
\hline Food from other places can expand your horizons & Get knowledge & \multirow[t]{3}{*}{ Information value } \\
\hline Practical cooking process & Acquire skills & \\
\hline The inclination of highly authentic & Authenticity & \\
\hline The inclination of funny content & Interestingness & \multirow[t]{3}{*}{ Entertainment value } \\
\hline Kill time & Kill time & \\
\hline Feel like eating food by self & Sensory impact & \\
\hline Calmness is good for sleep & Release pressure & \multirow[t]{2}{*}{ Emotional value } \\
\hline Feel happy when hungry & Positive emotion & \\
\hline Love to watch videos with culturally characteristic & Cultural connotation & \multirow[t]{2}{*}{ Symbolic value } \\
\hline $\begin{array}{l}\text { Love uploaders with a great attitude towards life, which is } \\
\text { consistent with my own values }\end{array}$ & Self identification & \\
\hline Videos with barrages are interactive & Interactive quality & Interaction degree \\
\hline
\end{tabular}

\subsection{VARIABLE DEFINATION AND HYPOTHESIS STUDY}

Information value refers to that users obtain information from food videos, learn relevant skills and knowledge, and believe that the information in the video is true and reliable [10]. Fan Xiaochun (2015) believes that new needs of users can be stimulated by improving the value of information [11]; Li Enke et al. (2016) believe that information value variables have a significant impact on the use intention of university mobile libraries [12]. Accordingly, the following assumptions are put forward.

\section{H1: The information value of food videos has a}

positive impact on users' willingness to interact.

Entertainment value means that users can spend their leisure time by watching food videos, which can bring users good sensory experience and happy feeling. Liu Dan (2011) and others analyzed the factors affecting users' acceptance of mobile advertising in Japan and Australia, and found that entertainment and credibility are the most important factors [13]; Yang Wei et al. (2016) discussed the impact of virtual community function on online purchase intention and found that community entertainment function has the greatest impact on online purchase intention [14]. Accordingly, the following assumption is put forward.

H2: The entertainment value of food videos has a positive impact on users' willingness to interact.

Emotional value refers to that food videos can relax the mood and enable users to obtain emotional experience and resonance. Zhao Linyu (2018) pointed out that emotional needs are one of the most important motivations in the use motivation of Cat's Kitchen [15]; Dong Yuhan (2021) mentioned that one of the reasons why BiliBili food videos are popular is that they can meet the emotional needs of a lonely society [16]. Accordingly, the following assumption is put forward.

H3: The emotional value of food videos has a positive impact on users' willingness to interact.

Symbolic value refers to the user's self-identity and personalized experience, and the self media style is consistent with the self perceived image. Today's food videos not only share food, users can also experience the cultural connotation of different regions and feel the life attitude and values of uploader in the food videos. Li Ziqi becomes popular because of the pastoral culture and in his video. Accordingly, the following assumption is put forward.

H4: The symbolic value of food videos has a positive impact on users' willingness to interact.

The degree of interaction refers to the degree to which the user's use intention and behavior are affected by other people and the environment. Chen Shu et al. (2017) pointed out that the influence and interaction of users affect people's willingness to forward microblogs [17]; Dong Yuhan (2021) pointed out that one of the reasons why BiliBili food videos are popular is the interactivity of barrage social networking. In order to better integrate users into the group, the evaluation of others and the popularity of we media imperceptibly affect users' willingness to interact.

H5: The interaction degree of gourmet videos has a positive impact on users' interaction intention.

The user interaction in BiliBili studied in this paper mainly refers to the user's behavior of exchanging information with each other, such as online reply, forwarding, comment, like, collection, sending barrage and so on. 


\section{EMPIRICAL RESEARCH DESIGN}

Considering the stability of measurement and the effectiveness of measurement results, the author refers to the mature scales of domestic scholars in related fields, and designs a gauge for this research content.
This study adopts the way of filling in the questionnaire online and publishing it through the questionnaire star. After 15 days of distribution, 284 questionnaires were sent out and 251 valid questionnaires were received, accounting for $88.38 \%$ of the total number of questionnaires.

Table 2 Descriptive Statistics of Scale Variables

\begin{tabular}{|l|l|l|l|l|}
\hline Variable & Minimum & Maximum & Average & Standard Deviation \\
\hline Information Value & 1.00 & 5.00 & 4.01 & 0.74 \\
\hline Entertainment Value & 1.00 & 5.00 & 4.12 & 0.68 \\
\hline Emotional Value & 1.00 & 5.00 & 4.08 & 0.75 \\
\hline Symbolic Value & 1.00 & 5.00 & 4.03 & 0.75 \\
\hline Degree of Interaction & 1.00 & 5.00 & 3.95 & 0.88 \\
\hline User Interaction Intention & 1.00 & 5.00 & 3.95 & 0.83 \\
\hline
\end{tabular}

\section{DATA PROCESSING AND ANALYSIS}

\subsection{DESCRIPTIVE STATISTICS}

For the basic information, it is found that young women with high education are the main users of food videos; Most users tend to interact; The proportion of watching food video for 1-2 years is the largest, accounting for $36 \%$, indicating that food video is still a developing field; $82 \%$ of the people don't have a favorite uploader, indicating that only a small number of uploaders have achieved personal brand accumulation; As for the frequency of watching food videos, the largest proportion is $48 \%$ on average once a week, indicating that users haven't formed a strong dependence on food videos. About the reasons for watching food videos, $70 \%$ of people are for entertainment and $51 \%$ are to meet the sense of intimacy and curiosity. This result implies that people will pay more attention to the entertainment and emotion of videos, which is very enlightening for the later research.

The second part of the questionnaire uses Likert's five level scale, giving 1-5 points from "completely disagree" to "fully agree", and each variable has 3-4 items. As shown in Table 2, the standard deviation of all variables is within 1 , and there is no abnormal value in the data. The average value can be used to describe each variable. The average value of each variable exceeds 3.5 , and most users have a good experience in these six dimensions during viewing.

\subsection{RELIABILITY ANALYSIS OF FORMAL SURVEY DATA}

Reliability is measured by Cronbacht a with the reliability analysis of the total sample, and the value of a coefficient is 0.946 .

The reliability of each variable is analyzed one by one. The values of reliability coefficients are greater than 0.7 , indicating that the data reliability quality of each variable is high.

\subsection{VALIDITY ANALYSIS OF FORMAL SURVEY DATA}

(1) Content validity. The scale of this study is formed on the basis of previous research results. The expression of items can be understood, and the content validity of the scale is good.

(2) Construct validity. Exploratory factor analysis is a commonly used structural validity analysis method in academic circles. The premise of using this analysis method is to judge whether the variable is suitable for factor analysis by butterball testing KMO value.

For the independent variable, the KMO value of the sample is 0.930 , and the $\mathrm{P}$ value corresponding to Barthes spherical test is 0.000 , so it can be determined that the data is suitable for factor analysis 。

Through factor analysis, five main factors are obtained, and the cumulative variance contribution rate of the factors is $68.930 \%$, and the index reaches $60 \%$, indicating that the main factors can explain the research content strongly.

Then the factor load analysis is carried out. According to the division of factor load coefficient after rotation, after deleting unsuitable items, the absolute value of factor load coefficient of each index is higher than 0.5 , and the aggregation validity of each factor is very good, which meets the research requirements.

For the dependent variable, the KMO value of the sample is 0.930 , and the $\mathrm{P}$ value corresponding to Barthes spherical test is 0.000 , so it can be determined that the data is suitable for factor analysis.

Then conduct factor analysis to obtain a main factor, but the cumulative variance contribution rate of the factor is $56.363 \%$, which is lower than $60 \%$. If the item with the smallest load value is deleted, the cumulative variance contribution rate of the factor is $60.532 \%$, which is greater than $60 \%$, which meets the research requirements. 
Then factor load analysis was carried out. The absolute values of factor load coefficients of each index were greater than 0.5 , indicating with good validity. to analyze the correlation between variables, and the correlation analysis results are obtained, as shown in Table 3.

\subsection{CORRELATION ANALYSIS}

In this paper, Pearson correlation coefficient is used

Table 3 Correlation Analysis Results of Each Variable

\begin{tabular}{|l|l|l|l|l|l|l|}
\hline Variable & $\begin{array}{l}\text { Information } \\
\text { Value }\end{array}$ & $\begin{array}{l}\text { Entertainment } \\
\text { Value }\end{array}$ & $\begin{array}{l}\text { Emotional } \\
\text { Value }\end{array}$ & $\begin{array}{l}\text { Symbolic } \\
\text { Value }\end{array}$ & $\begin{array}{l}\text { Degree of } \\
\text { Interaction }\end{array}$ & $\begin{array}{l}\text { User } \\
\text { Interaction } \\
\text { Intention }\end{array}$ \\
\hline Information Value & 1 & & & & & \\
\hline Entertainment Value & $0.342^{* *}$ & 1 & & & & \\
\hline Emotional Value & $0.412^{* *}$ & $0.612^{* *}$ & 1 & & & \\
\hline Symbolic Value & $0.491^{* *}$ & $0.470^{* *}$ & $0.552^{* *}$ & 1 & & \\
\hline Degree of Interaction & $0.460^{* *}$ & $0.417^{* *}$ & $0.522^{* *}$ & $0.583^{* *}$ & 1 & \\
\hline User Interaction Intention & $0.524^{* *}$ & $0.475^{* *}$ & $0.610^{* *}$ & $0.617^{* *}$ & $0.546^{* *}$ & 1 \\
\hline
\end{tabular}

$* \mathrm{p}<0.05 * * \mathrm{p}<0.01$

In this study, the $\mathrm{P}$ value corresponding to the correlation coefficient of all variables is less than 0.01 , indicating that all variables are closely related, and the correlation coefficient values are greater than 0 , indicating that there is a positive correlation. Then compare the value of correlation coefficient. In the relationship between the five variables and user interaction intention, the correlation from strong to weak is symbolic value $>$ emotional value $>$ interaction degree $>$ information value $>$ entertainment value.

\subsection{RRGRESSION DATA ANALYSIS}

This paper uses multiple regression analysis to explore the regression analysis between each dimension of BiliBili food video and user interaction intention.
Taking the five factors of BiliBili food video, information value, entertainment value, emotional value, symbolic value and interaction degree as independent variables and user interaction intention as dependent variables for regression analysis, it is found that the $\mathrm{P}$ value corresponding to entertainment value in the model is greater than 0.05 , indicating that the influence relationship between entertainment value and user interaction intention is not significant, and $\mathrm{H} 2$ is rejected. The reason is that two items in the entertainment value are deleted because they don't meet the validity analysis, while the remaining items pay more attention to interest and freshness, and these two items have no significant impact on users' interaction intention. Delete the entertainment value and repeat the above operation. The analysis results are shown in Table 4 .

Table 4 Linear Regression Analysis Results between Each Dimension of BiliBili Food Video and User Interaction Intention after Optimization $(\mathrm{n}=251)$

\begin{tabular}{|c|c|c|c|c|c|c|c|c|}
\hline \multirow[t]{2}{*}{ Variable } & \multicolumn{2}{|c|}{$\begin{array}{l}\text { Non-standardized } \\
\text { Coefficient }\end{array}$} & $\begin{array}{l}\text { Standardized } \\
\text { Coefficient }\end{array}$ & $\mathrm{p}$ & VIF & $\mathrm{R}^{\wedge} 2$ & $\begin{array}{l}\text { Adjustment } \\
\mathrm{R}^{\wedge} 2\end{array}$ & $\mathrm{~F}$ \\
\hline & $\mathrm{B}$ & $\begin{array}{l}\text { Standard } \\
\text { Error }\end{array}$ & Beta & & & 0.536 & 0.526 & $\begin{array}{l}F=56.586 \\
p=0.000\end{array}$ \\
\hline Constant & 0.451 & 0.426 & & 0.291 & & & & \\
\hline Information Value & 0.334 & 0.087 & 0.201 & $0.000 * *$ & 1.430 & & & \\
\hline Entertainment Value & 0.108 & 0.107 & 0.057 & 0.312 & 1.677 & & & \\
\hline Emotional Value & 0.543 & 0.119 & 0.281 & $0.000 * *$ & 1.990 & & & \\
\hline Symbolic Value & 0.491 & 0.113 & 0.260 & $0.000 * *$ & 1.891 & & & \\
\hline Degree of Interaction & 0.215 & 0.093 & 0.132 & $0.022 *$ & 1.736 & & & \\
\hline
\end{tabular}

Dependent variable: $D-W$ value of user interaction intention : $1.690 * p<0.05 \quad * * \quad p<0.01$

(1) The P values corresponding to each variable in the model are less than 0.05 , indicating that the influence relationship between independent variables and dependent variables is significant.

(2) The VIF values of all variables in the model are less than 5 , indicating that there is no collinearity problem, indicating that the model is good.

(3) The R-square value of the model is 0.534 , which means that the information value, emotional value, symbolic value and interaction degree can explain 53.4\% of the reasons for the change of purchase intention, indicating that the fitting degree of the model is high.

(4) Through b-value analysis, the influence relationship between variables: the regression coefficient values of information value, emotional value, symbolic value and interaction degree of the four variables are positive, indicating that the independent variable has a 
positive impact on the dependent variable. The influence of independent variables on dependent variables is: emotional value $>$ symbolic value $>$ information value $>$ interaction degree. The analysis results verify the research hypotheses $\mathrm{H} 1, \mathrm{H} 3, \mathrm{H} 4$ and $\mathrm{H} 5$. Therefore, the multiple linear regression equation can be obtained: Purchase intention $=0.566+0.338^{*}$ information value $+0.596^{*}$ emotional value $+0.507^{*}$ symbolic value $+0.220 *$ interaction degree.

\section{CONCLUSION}

\subsection{RESEARCH CONCLUSION}

Among the five hypotheses proposed in the research, $\mathrm{H} 1, \mathrm{H} 3, \mathrm{H} 4$ and $\mathrm{H} 5$ are set up, $\mathrm{H} 2$ is not.

Therefore, the conclusion of this study is as following.

(1) In the process of watching BiliBili food video, the information value, emotional value, symbolic value and interaction degree of food video will significantly and positively affect users' interaction intention. In this questionnaire survey, entertainment value doesn't significantly affect users' willingness to interact.

(2) The influence of food video factors on users' interaction intention is emotional value $>$ symbolic value $>$ information value $>$ interaction degree, that is, emotional value has the greatest influence on users' interaction intention, followed by symbolic value and information value, and the factor with the least influence is interaction degree.

\subsection{MANAGEMENT SUGGESTIONS}

Suggestions for food video uploaders: (1) Establish personal brand and enhance popularity. Uploaders should pay attention to their own advantages, give play to their own characteristics, accumulate fans, establish a personal brand and improve popularity. (2) Pay attention to the emotional expression and connotation of food videos. Uploaders should meet the emotional needs of the audience through sound, copywriting and plot, arouse the emotional resonance of users, and display positive life attitudes and values. (3) Cultivate trust. Uploaders can provide users with more real experience and practical suggestions by making delicious food or displaying delicious food in a deeper and more grounded way, and actively interacting with users.

Suggestions for we media platform: (1) Increase the promotion of food uploaders. In this questionnaire survey, there are still some users who have not been exposed to food videos, and the platform can be moderately promoted. (2) Introduce good policies to support highquality food uploaders. There are many food uploaders, but few are famous and have a certain brand effect. The platform needs to introduce some policies to support those uploaders with strong professional ability and personal characteristics, meet users' personalized and diverse needs, and improve users' experience of watching food videos.

Suggestions for users: (1) Correctly understand their own needs and distinguish the authenticity and rational consumption in the process of watching videos. (2) Select an uploader with high-quality content. Because the background and ability of uploader are mixed, users should choose uploaders with strong personal ability, high-quality content and personal characteristics to watch. (3) Appropriately control the length and frequency of watching food videos. Most of the people who watch food videos are college students or young white-collar workers. They are in the stage of learning or career struggle. They should cherish their time and study hard to improve their skills.

\section{REFERENCES}

[1]Cao Xiaojing. BiliBili From A Small Subculture to the Mainstream [J].Journal of News Research, 2017,8(13):43+97.

[2]Huoshaoyun Data[DB/OL]. http://www.hsydata.com/dataAdviser/boardData, 2021-08-02/2021-08-02

[3]Zheng Shengying. On the Development of Food Short Video under the Trend of Vertical Content Segmentation [J].North Media Research,2017(4):64-67.

[4]Tang Menglin. Content Characteristics and Existing Problems of Short Food Videos [J]. Youth Journalist, 2018(2):90-91.

[5]Huang Lili. Symbolic Meaning Construction of Short Food Videos In The Context of Consumption Culture -- Taking "Li Ziqi" as an Example [J]. New Media Research, 2019,5(7):117-119.

[6]Weng Lvgang. Research on User Experience Guided By Interactive Behavior [D]. Wuxi:Jiangnan University,2009.

[7]Luo Huanyi. On The Interactivity of Network Video under the background of media integration [D]. Guangzhou:Jinan University,2015.

[8]Wang Xinwei,Li Shimeng,Wangnan Axue, Yang Mengqing. Research on Influencing Factors of Consumers' Information Interaction Willingness in the New Media Environment: An Example of the Automobile Industry New Media Platform [J]. Library and Information Service.,2017,61(15): 1524.

[9]Fan Xiaochun. An Empirical Study on The Influencing Factors of Users' Willingness To Use Behavior in Mobile Library --- An Analysis From The 
Perspective of Information Ecology [J].Researches on Library Science, 2015(14) : 85-93.

[10]Li Enke,Xu Qiang,Guo Lujie. Empirical Research on the Influencing Factors of Users' Intention to Adopt University Mobile Library [J].Library Tribune, 2016,36(1):85-93.

[11]Liu Dan,Zhang Jing.TAM-based study on factors influencing the adoption of mobile payment [J].China communications, 2011(3):198-204.

[12]Yang Wei,Sheng Yuhua,Xu Zhaojun. Virtual Community Function and Online Purchase Intention Based on Personality Traits --- An Empirical Study from the Perspective of Interaction [J].Enterprise Economy,2016(4):102-106.

[13]Zhao Linyu. Research on Communication Strategy Based on User Psychology --- Taking @ Rishiji as an Example [D].Chengdu:Southwest Jiaotong University,2018.

[14]Dong Yuhan. Analysis on the Popularity of BiliBili Food Videos [J].Voice and Screem World, 2021(3):58-59.

[15]Chen Shu,Dou Yongxiang,Zhang Qingjie. Research on the Influential Factors of the Reposting Behavior of Microblog Users Based on the Theory of Reasoned Action[J].Journal of Intelligence, 2017,36(11): 147-152+160. 